\title{
Pearls in Clinical Evaluation and Office Management of Patients with Synkinesis
}

\author{
Shiayin Yang $^{1}$ and Jennifer Kim ${ }^{2}$ \\ ${ }^{1}$ Vanderbilt University Medical Center \\ ${ }^{2}$ University of Michigan
}

May 6, 2020

\begin{abstract}
Facial synkinesis is the long-term sequela of facial nerve injury that can result in severe deficits in facial expression and function. It is characterized by abnormal involuntary facial movements that accompany voluntary facial expressions. The type and severity of symptoms differ from patient to patient, which makes evaluation and management complex. Given the chronicity of the disease, appropriate evaluation is paramount to effective management. This article will discuss how to effectively evaluate patients in the office and associated treatment.
\end{abstract}

Introduction

Facial synkinesis is a sequela of facial nerve injury characterized by the abnormal involuntary movement of facial musculature that accompanies voluntary facial movement. There is often associated hyperkinesis and contracture of the facial muscles. The most common etiology is Bell's palsy of which over $70 \%$ of patients have complete recovery of facial nerve function. The remaining $30 \%$ of patients have incomplete recovery, which can result in functional or aesthetic deficits. ${ }^{1}$ The incidence of post-paralysis synkinesis from other etiologies ranges between $15-20 \% .^{2,3}$

Different theories have been proposed to explain the pathogenesis of synkinesis. Aberrant nerve regeneration is one of the most accepted theories. This theory hypothesizes that after nerve injury, there is nonspecific axon regeneration and misdirected rerouting of nerve fibers resulting in abnormal muscle contraction with voluntary facial movement. Other theories have postulated reorganization and hypersensitization of the facial nucleus. ${ }^{1,4,5}$

Synkinesis can be extremely debilitating from both a functional and psychosocial standpoint. ${ }^{6}$ The spectrum of symptomatology is wide and varies from patient to patient. Appropriate management of synkinesis requires a systematic evaluation and individualized treatment plans. Injections with neuromodulators have been demonstrated to be an effective mainstay in the treatment. ${ }^{7}$ The purpose of this paper is to discuss the comprehensive in office evaluation a patient with synkinesis and management with neuromodulator therapy. The senior author uses botulinum toxin (Botox ${ }^{\circledR}$ ) as a neuromodulator but other types of neuromodulators can be used as well.

History 
When evaluating the synkinetic patient the provider should first establish the etiology of synkinesis - Bell's palsy, trauma, iatrogenic, congenital. Bell's palsy is one of the most common causes of synkinesis. It is then important to ascertain the timing since injury as the degree of synkinesis may worsen as more time elapses. Typically, the onset of synkinesis occurs 3-6 months after facial nerve injury and may progress up to 2 years. Another critical aspect to obtain during the history is the degree of the initial facial paralysis (complete versus incomplete) and the time interval between paralysis and recovery. Patients with incomplete paralysis and those who regain function quickly, tend to have improved prognosis compared to patients with complete facial paralysis and those who take longer to recover. The provider should ask about prior medical and surgical treatments as this is necessary in determining appropriate treatment plans and understanding what options are available to the patient. Lastly, the most important aspect of the history, is understanding what is most bothersome to the patient and what they would like to achieve with treatment. The provider may notice something on physical exam that they believe should be corrected, but to the patient this same finding is not bothersome or is of lower priority. Thus, open dialogue and shared decision-making about functional and aesthetic issues coupled with realistic treatment options are paramount and allows for shared decision making.

\section{Clinical Evaluation}

A detailed clinical examination is performed to evaluate facial symmetry and function at rest and with volitional movement. At rest, the entire face is evaluated to determine asymmetries between the affected (synkintetic) and unaffected (non-synkinetic) sides. The affected side can appear tight or contracted compared to the unaffected side. Assessment includes meticulous evaluation of the brow position, palpebral fissure height, dynamics of the eye blink, depth and orientation of the nasolabial fold, and position of the oral commissure (Figure 1). A thorough zonal assessment of the face is performed to gauge the degree and effect of synkinesis. At each exam, a systematic top-down approach is used to evaluate the following volitional facial movements: brow elevation, gentle and forceful eye closure, smile with and without teeth show, lip pucker, puffing out cheeks, depression of the lower lip, and platysmal banding (Figure 2). The degree of movement is documented as well as the presence and degree of ocular, midfacial, oral, mentalis, and platysmal synkinesis. Careful examination of these different movements is critical as it dictates location and amount of Botox ${ }^{\circledR}$ to be injected. In addition to volitional movements, it is important to evaluate patients at rest, while speaking and with a spontaneous smile.

\section{Upper Face}

Examination begins with the upper face with documentation of the brow at rest and elevation. A common clinical finding is an elevated brow at rest and poor activation of the frontalis on the affected side. The corrugator supercilii is evaluated by having the patient furrow their brow. Patients will demonstrate limited movement of the corrugator with activation but paradoxical elevation of the affected brow due to contraction of the frontalis and corrugator supercilii can sometimes be seen with forceful eye closure (Figure 3).

\section{Eyes}

Evaluation proceeds to the eyes with documentation of the palpebral fissure dimensions. In severely synkinetic patients, the palpebral opening of the affected eye tends to be narrower at rest due to hypercontraction of the orbicularis oculi. Forceful eye closure is used to assess the function of the orbicularis oculi and determine the presence of oculooral synkinesis. Gentle eye closure or light blinking is performed to evaluate synkinesis of the preseptal and pretarsal orbicularis oculi. Often hyperkinesis of the pretarsal and preseptal orbicularis increases with smile, pucker, and puffing out the cheek. At the same time, they can have slowed blink mechanism predisposing them to dry eye. Patients can also have increased tearing with eating.

\section{Midface}

Accurate evaluation of the midface can be challenging due to the multiple facial muscles involved in midfacial movement. At rest it is important to note the depth orientation of the nasolabial fold. A deepened nasolabial fold and midfacial bulk can be secondary to hyperkinesis of the zygomaticus major, zygomaticus minor, 
and levator labii superioris. Synkinesis of these muscles can limit the degree and vector of oral commissure excursion. Hypercontraction of the nasalis can cause constriction of the nostril leading to nasal obstruction. Overall, a patient's midface will be tightened compared to the unaffected side and they will often note pain and tightness.

Lower Face

Thorough examination of the oral commissure at rest and with movement is critical as the most common patient complaint is a lopsided smile or obvious asymmetries while speaking. At rest, it is important to note if the position of the oral commissure compared to the unaffected side. With smile, patients tend to have decreased excursion and less teeth show on the affected side. Multiple groups of muscles responsible for smile excursion insert and pull on the orbicularis in vertical, superolateral, lateral and inferior directions. Hyperkinesis and simultaneous activation of these muscles cancel out the vectors of pull on the modiolus resulting in poor excursion. Hyperkinesis of buccinator, depressor labii inferioris, and depressor anguli oris results in tethering of the oral commissure and prevents full upward excursion. The depressors are not paralyzed but rather hyperkinetic, which prevents normal relaxation and contraction resulting in limited excursion and lower teeth show. Patients will often avoid smiling due to the obvious asymmetries. The buccinator can be evaluated by having the patient close their eyes tightly while keeping their mouth open. With synkinesis of the buccinator, intraorally the buccinator will be more convex than concave and a tight band can be palpated. Patients may state that they bite their cheek on the affected side when eating. Evaluation of the orbicularis oris, lower lip depressors, and platysma can be accomplished by having the patient purse their lips and pronounce the letter "e". The lips on the affected side can appear shortened from cupids bow to the oral commissure due to orbicularis oris synkinesis and the lower lip may sit higher than the unaffected side due to depressor dysfunction.

Synkinesis of the mentalis and platysma can also contribute to irregularities in speech and smile. Findings of mentalis synkinesis include a peau d'orange appearance at rest and dimpling associated with other facial movements. Platysmal banding at rest and with movement is a common finding in patients with synkinesis. The posterior belly of the digastric muscle is another facial muscle that can be affected in synkinesis. It is difficult to evaluate this muscle on physical exam and the best indicator is based on the patient's history. Patients will complain of severe facial pain, pressure, or tightness and when asked to pinpoint the origin of the pain they localize to the insertion of the posterior belly of the digastric at the mastoid notch just behind the mandible.

Documentation

Photo documentation is essential to guide therapy. At each visit, photographs are taken of the patient performing each of the volitional facial movements described above. Video documentation is performed at the first office visit and when there is any change in physical exam from prior visits. Patients also complete the Facial Clinimetric Evaluation (FaCE) scale and Synkinesis Assessment Questionnaire (SAQ), which are self-reported instruments used to measure disease-specific quality of life as well as physical and psychosocial impairment.

\section{Treatment Options}

Successful treatment of synkinesis requires a multidisciplinary approach including physical therapy and medical therapy. Recently, novel surgical techniques for treatment of synkinesis have been described in the literature including transection of the affected muscles ${ }^{8-10}$ and selective neurectomy ${ }^{11-12}$, however, long-term data efficacy data is lacking. When a patient is evaluated in clinic for the first time they are counseled on treatment options with an understanding that successful treatment is comprised of multiple components.

Physical Therapy

All patients who present with synkinesis are also evaluated by a physical therapist who specializes in facial paralysis. Physical therapy has been shown to improve quality of life in patients with facial paralysis. ${ }^{13}$ 
Therapy techniques include neuromuscular retraining, massage, relaxation, and biofeedback. ${ }^{14}$ With neuromuscular retraining, patients learn how to isolate specific muscles and bring involuntary movement under conscious control in order to improve resting tone and expression. ${ }^{14-16}$ Physical therapy is initiated prior to the initiation of medical therapy and continued in coordination with medical therapy.

Botulinum Toxin Injection

Chemodenervation is an effective treatment for synkinesis in the clinical setting. ${ }^{17-18}$ In the senior author's practice, Botox ${ }^{\circledR}$ is the primary agent used unless contraindicated or if it is believed the patient may have developed antibodies to Botox ${ }^{\circledR}$. Prior to the initiating therapy, it is important that the patient understands that selective chemodenervation is not an exact science and that it may take several trials to establish the optimal treatment plan. It is important that the patient understands their condition is not reversible and that our goal of therapy is to improve facial function, symmetry, and discomfort.

The amount and location injected varies by patient. However, the general approach that is used for new patients will be described. A solid understanding of facial musculature is critical in order to accurately target the affected muscles. Once the face has been fully examined and photographs taken, topical lidocaine may be applied to the patients face and allowed to sit for 10 minutes. Additional items that can be used to help lessen injection site pain include ice and ShotBlocker ${ }^{\circledR}$ device. Similar to the clinical examination, Botox ${ }^{\circledR}$ injection is performed in a systematic manner. The senior author will treat both the affected and unaffected sides with botox in order to obtain appropriate facial symmetry.

\section{Upper Face}

Beginning with the forehead, 5-10 units are injected into the unaffected frontalis muscle and 7.5-12 units injected into the corrugator to improve symmetry of the forehead by decreasing the amount of animation on the unaffected side. At rest, the brow on the affected side can be positioned higher than the unaffected brow. This asymmetry can be treated by injecting 5-10 units into the affected frontalis muscle to relax the elevated brow of the affected side and address the synkinetic elevation with eye closure or injecting 4-6 units to target and relax the outer orbicularis oculi at the level of the brow in order to raise it. If the patient demonstrates synkinetic contraction of the corrugator and procerus with forceful eye closure, this can be treated with 7.5 - 12 units of Botox ${ }^{\circledR}$. See Figure 4 for injection sites of the upper face. The senior author finds it important to treat both sides of the upper face in order to obtain appropriate symmetry at rest and with movement.

\section{Eyes}

Given ocular synkinesis is a common presenting complaint, Botox ${ }^{\circledR}$ therapy is an essential aspect in management. When initiating therapy, it is important to begin conservatively as aggressive treatment can result in slowed eye blink and lagophthalmos, which places the patient at risk for exposure keratopathy. It is important to evaluate the pretarsal, preseptal, and outer orbicularis oculi in order to determine the affected muscle responsible for the patient's complaints. For patients with a narrowed palpebral fissure at rest, injection of the outer orbicularis oculi and lateral orbicularis oculi with $8-12$ units of Botox ${ }^{\circledR}$ is used to improve palpebral opening. This injection can also be used to counteract the degree of ocular synkinesis. If the patient demonstrates severe ocular synkinesis, injection of the pretarsal orbicularis oculi can be performed with 1-2 unit of Botox ${ }^{\circledR}$. Often it is activation of the pretarsal or preseptal orbicularis oculi that is the responsible for constriction of the palpebral fissure and thus, it is important to evaluate and treat these muscles. It is critical to assess if it's the upper eyelid or lower eyelid responsible for synkinesis. This can be down by evaluating the lid margin level and change in relation to the iris. It is safer to begin treatment of the lower eyelid as there is a lower risk of causing dry eyes. The unaffected side is typically left untreated but lateral orbicularis oculi can be treated for symmetry. See Figure 4 for injection sites of the eye.

\section{Midface}

Patients with a deepened nasolabial fold and significant midfacial bulk and tightness can be treated with 1-2 units to the zygomaticus major. The nasalis, zygomaticus minor and levator labii superioris can also be treated with 1-2 units of Botox ${ }^{\circledR}$ for deepened nasolabial folds. Caution should be taken with these injections 
as over-injection can drop the upper lip and decrease the amount of upward excursion with smile. See Figure 4 for injection sites of the midface.

Lower Face

Treatment of the lower face can be complex and depends on the cosmetic and functional deformity. Often times, the affected side has restricted upward oral commissure excursion, a downward oral commissure position at rest, and decreased teeth show with smiling. In order to improve oral commissure excursion, it is important to take into consideration all of these aspects. To improve upward excursion of the oral commissure, $2-5$ units of Botox ${ }^{\circledR}$ can be injected into the buccinator. ${ }^{19}$ Conservative injection is recommended as over injection can result in difficulties eating and speaking. To improve the oral commissure position at rest, both the depressor anguli oris and the insertion of the platysma along the mandible can be targeted with 1-2 units and 5-10 units of Botox ${ }^{\circledR}$ respectively. Often the contralateral nonaffected depressor is hyperactive and patients may complain of lower lip biting of the affect side resulting from unopposed pull. Conservative injection with 1-2 units of Botox ${ }^{\circledR}$ to the unaffected depressor is often helpful. Platysmal banding can be injected with $10-20$ units of Botox ${ }^{\circledR}$. Treatment of mentalis synkinesis can be accomplished with $10-15$ units (refer to Figure 4). Injection of the posterior belly of the digastric can be used to treat posterior neck pain or tightness, which is often described as spasm brought on with laughing, yawning, or chewing. The posterior belly of the digastric can be identified using anatomic landmarks or ultrasound and it is treated with $10-15$ units of Botox ${ }^{\circledR}$.

Local Nerve Blocks

Local nerve blocks are a diagnostic test that can be performed in the office to identify nerves contributing to synkinetic movement. Blocks are performed with a 1:1 mixture of 1\% lidocaine with 1:100,000 epinephrine and $0.25 \%$ bupivacaine. Injection with local anesthetic temporarily blocks nerve conduction and results in paralysis of the muscle it innervates. This test is a valuable asset in determining if a patient is a candidate for selective neurectomy and can be helpful as a test prior to injecting Botox ${ }^{\circledR}$ (see Figure 5). The location of injection of the nerve block depends on the patient's complaints. If the patient has significant platysmal banding and neck tightness, the cervical branch of the facial nerve can be targeted with 1-2 $\mathrm{mL}$ of the local mixture. If the patient reports limited oral commissure excursion or tethering of their smile, the marginal mandibular branch can be targeted with $1-2 \mathrm{~mL}$ of the local mixture. If the patient reports facial tightness, cheek biting, or issues with eating, the buccinator can be targeted with 1-2 $\mathrm{mL}$ of the local mixture. Often combinations of the above blocks are used starting first with a cervical branch block then proceeding to marginal mandibular branch block and then to a buccinator block. Patient's are evaluated after each block by having them perform the above described facial expressions and subjective improvement of symptoms. The effects of the injections wear off after a couple of hours and patients are asked to document how the injections improved/worsened their symptoms. Patients are evaluated multiple times and different nerve blocks trialed prior to consideration of surgery. Patients who are considered candidates for selective neurectomy are those with longstanding, debilitating synkinesis requiring significant Botox ${ }^{\circledR}$ injections and who have consistently noted benefit from injection of local anesthetics.

Follow-Up

New patients are seen one month after injection with Botox ${ }^{\circledR}$ to evaluate the effectiveness of their initial treatment and determine if more treatment is needed. Return patients are seen every $3-4$ months for repeat injections. Meticulous documentation of the amount and site of Botox ${ }^{\circledR}$ injected as well as photographic and video documentation are essential and allows the clinician to appropriately tailor treatment to each patient and identify areas of improvement.

References:

1. Peitersen E. The natural history of Bell's palsy. Am J Otol 1982;4(2):107-111.

2. Moran CJ, Neely JG. Patterns of facial nerve synkinesis. Laryngoscope 1996;106:1491-96. 
3. Celik M, Forta H, Vural C. The development of synkinesis after facial nerve paralysis. Eur Neurol 2000;43:147-151.

4. Crumley RL. Mechanisms of synkinesis. Laryngoscope 1979; 89:1847-54.

5. Takeda T, Takeda S, Okada T, et al. Experimental studies on the recovery processes from severe facial palsy and the development of its sequelae. Otol Neuro 2015;36(5):896-903.

6. Neely JG, Neufeld PS. Defining functional limitation, dis-ability, and societal limitations in patients with facial paresis: Initial pilot questionnaire. Am J Otol 1996;17:340-342.

7. Filipo R, Spahiu I, Covelli E, et al. Botulinum toxin in the treatment of facial synkinesis and hyperkinesis. Laryngoscope 2012; 122:266-270.

8. Labbe D, Benichou L, Iodice A, et al. Depressor anguli oris sign (DAO) in facial paresis: how to search it and release the smile. Ann Chir Plast Esthe 2012; 57:281-285.

9. Lindsay RW, Edwards C, Smitson C, et al. A systematic algorithm for management of lower lip asymmetry. Am J Otolaryngol 2011; 32:1-7.

10. Henstrom DK, Malo JS, Cheney ML, et al. Platysmectomy: an effective intervention for facial synkinesis and hypertonicity. Arch Facial Plast Surg 2011;13:239-243.

11. Hohman MH, Lee LN, Hadlock TA. Two-step highly selective neurectomy for refactory periocular synkinesis. Laryngoscope 2013; 123:1385-88.

12. Azizzadeh B, Irvine LE, Diels J, et al. Modified selective neurectomy for the treatment of post-facial paralysis synkinesis. Plast Reconstr Surg 2019; 143(5):1483-96.

13. Luijmes RE, Pouwels S, Beurskens CHG,et al. Quality of life before and after \& different treatment modalities in peripheral facial palsy: a systematic review. Laryngoscope 2017; 127:1044-1051.

14. Diels HJ, Beurskens C. Neuromuscular retraining: nonsurgical therapy for facial nerve palsy. The Facial Nerve. New York, NY: Thieme; 2014; 205-212.

15. Balliet R, Shinn JB, Bach-y-Rita P. Facial paralysis rehabilitation: retraining selective muscle control. Int Rehabil Med 1982; 4:67-74.

16. Cronin GW, Steenerson RL. The effectiveness of neuromuscular facial retraining combined with electromyography in facial paralysis rehabilitation. Otolaryngol Head Neck Surg 2003; 128:534-538.

17. Mehta RP, Hadlock TA. Botulinum toxin and quality of life in patients with facial paralysis. Arch of Facial Plast Surg 2008 Mar 1;10:84-7.

18. Choi KH, Rho SH, Lee JM, et al. Botulinum toxin injection of both sides of the face to treat postparalytic facial synkinesis. J Plast Reconstr Aesthet Surg 2013 Aug 1;66(8):1058-63.

19. Wei LA, Diels J, Lucarelli MJ. Treating buccinator with botulinum toxin in patients with facial synkinesis. Ophthalmic Plast Reconstr Surg 2016;32(3):138-41.

Figure 1 - Evaluation of patient with synkinesis at rest.

Figure 2 - Volitional movements (brow elevation, gentle and forceful eye closure, smile with and without teeth show, lip pucker, and lower lip depressor) evaluated at each clinical visit.

Figure 3 - Paradoxical elevation of the brow on the affected side from contraction of the frontalis and corrugator supercilia with forceful eye closure.

Figure 4 - General distribution of botulinum toxin injection in a patient with synkinesis.

Figure 5 - Patient with synkinesis prior to injection with lidocaine (a). Patient after injection of lidocaine of her right marginal mandibular nerve (b) and after injection of right marginal mandibular nerve and right buccal nerve (c), resulting in greater oral commissure excursion and smile symmetry. 


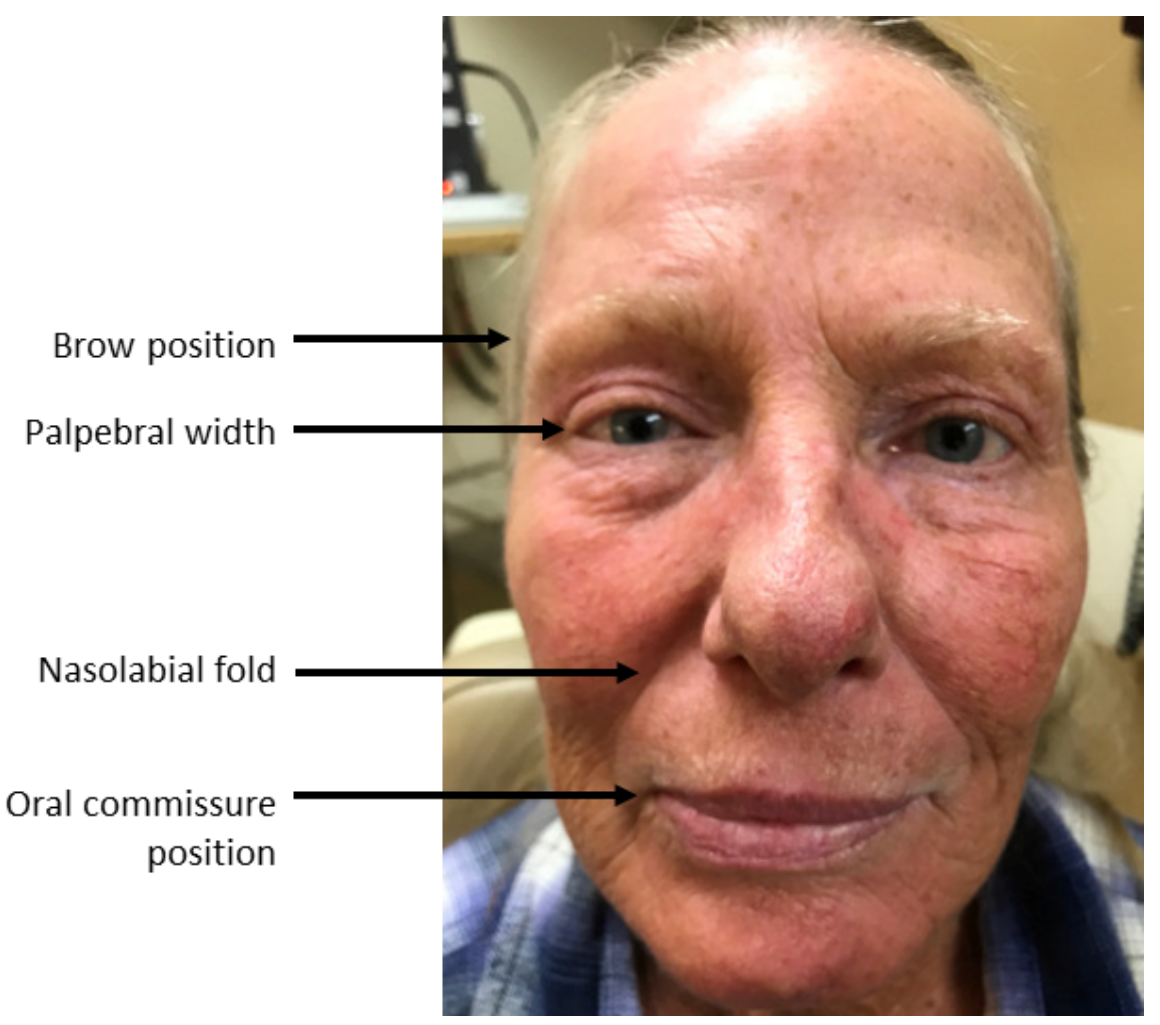



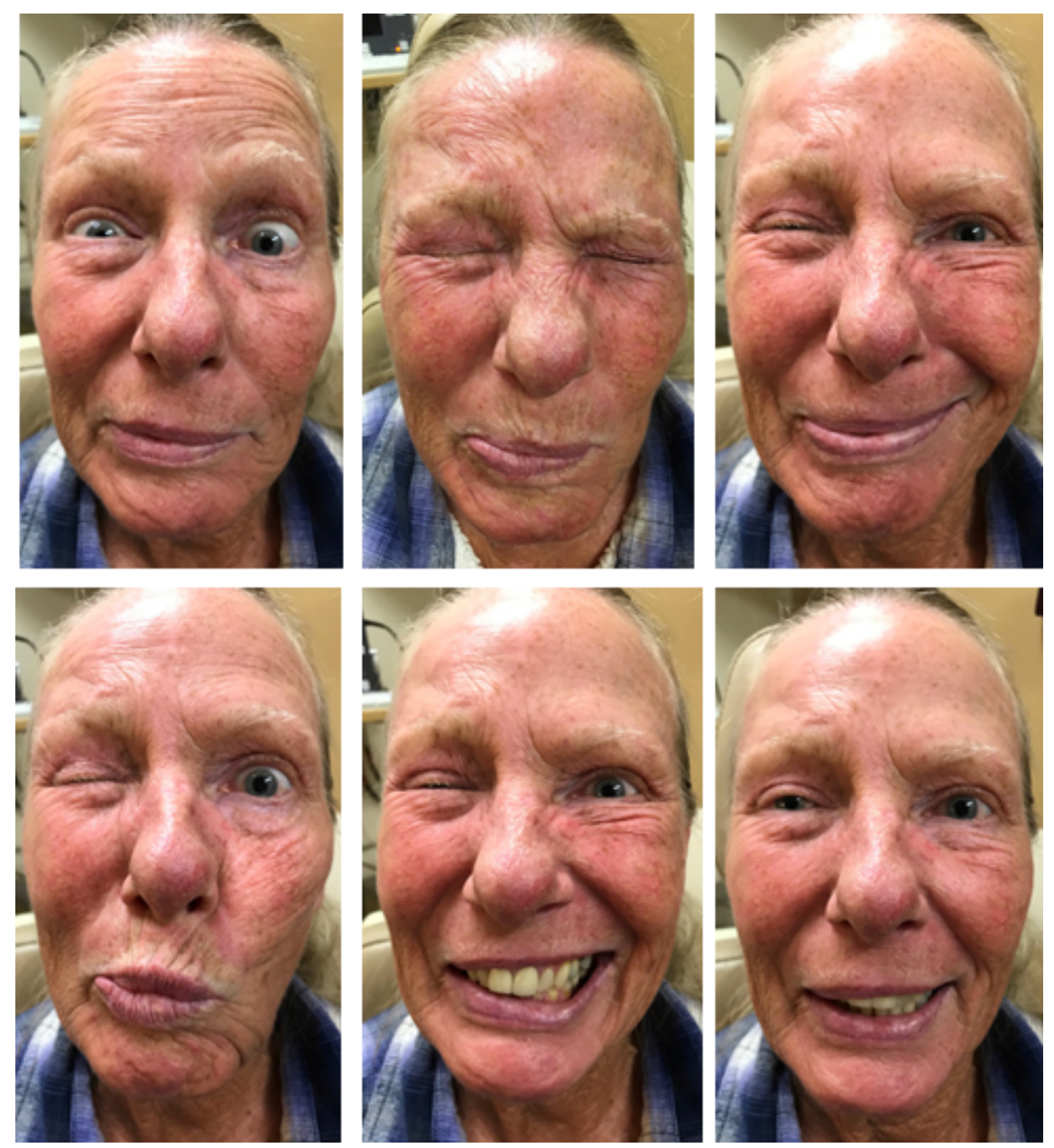


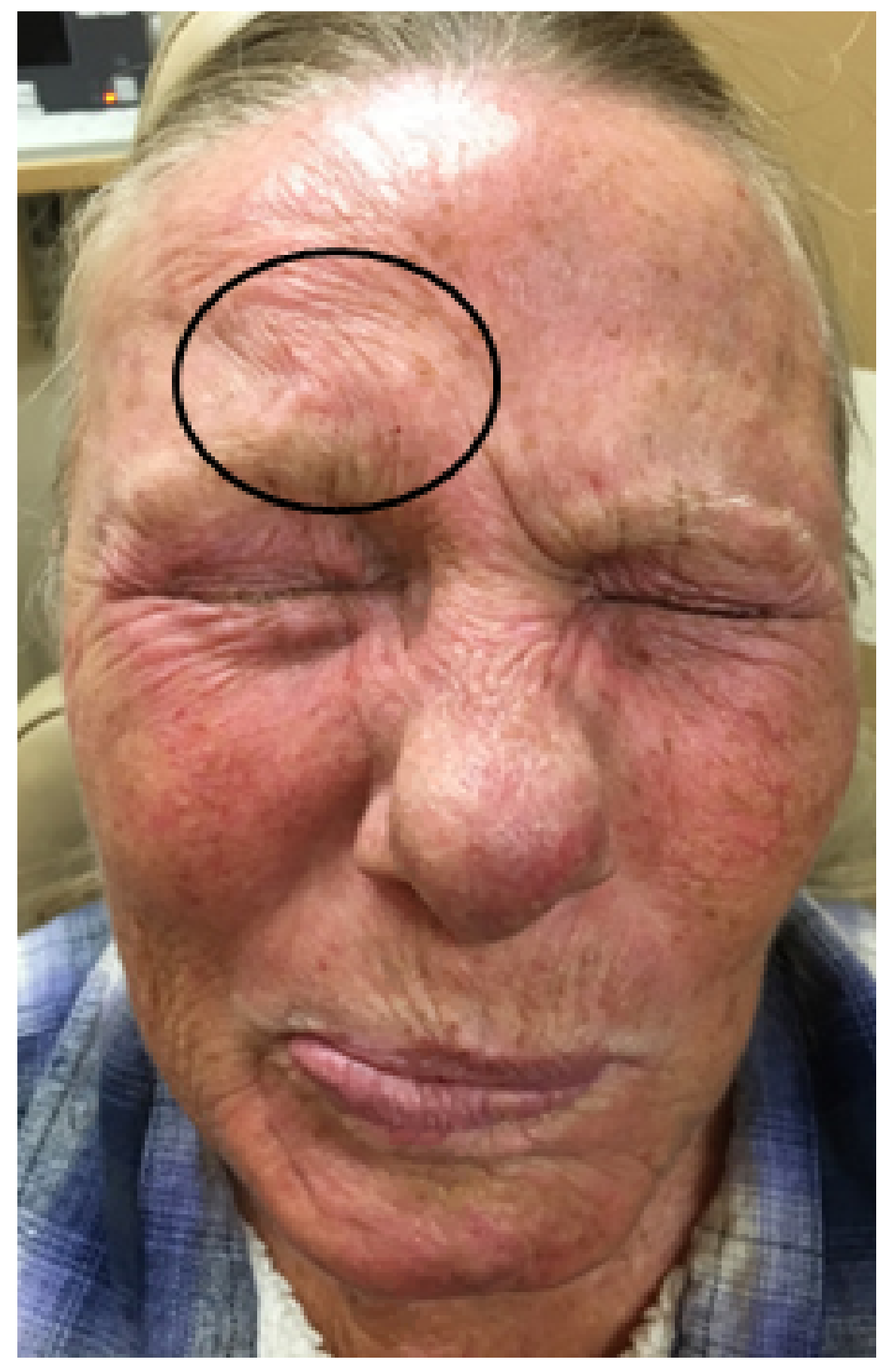



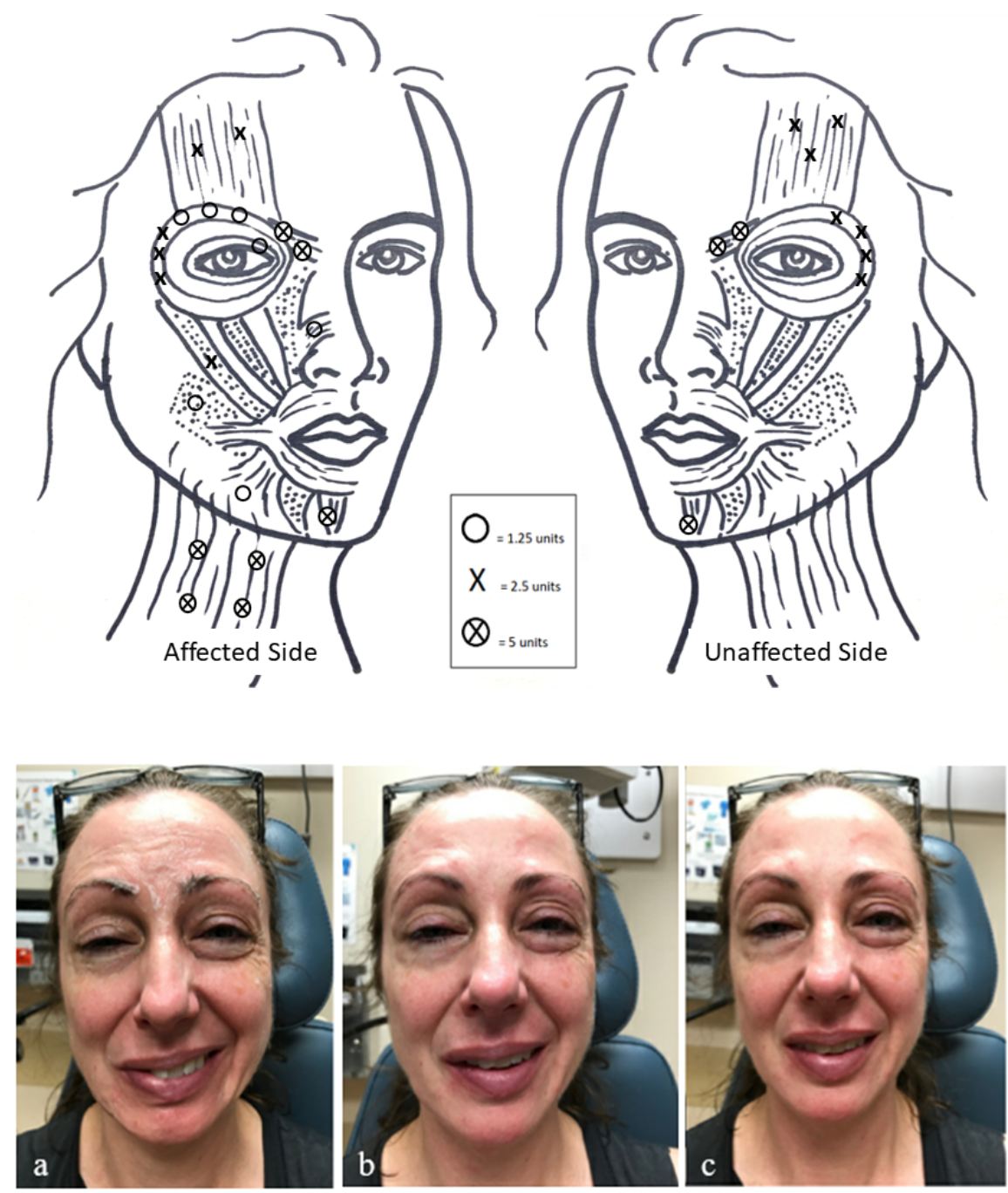